\title{
UNIFORMLY CONTRACTIVE FLXED POINTS IN COMPACT METRIC SPACES
}

\author{
SOLOMON LEADER
}

\begin{abstract}
Let $f$ be a continuous self-map on a compact metric space. Equivalent conditions are given for the existence of a uniformly contractive fixed point $w\left(f^{n} x \rightarrow w\right.$ uniformly for all $x$ ). Corollaries give sufficient conditions for uniformly contractive fixed points subsuming some known results.
\end{abstract}

1. Introduction and theorem. Let $f$ be a continuous self-map on a Hausdorff space $X$. Let $N$ be the set of all positive integers. A point $w$ in $X$ attracts a subset $A$ of $X$ if

$$
f^{n} x \rightarrow w \text { as } n \rightarrow \infty \text { in } N
$$

uniformly for all $x$ in $A$. For $A$ nonempty (1) implies $f w=w$ by continuity. We say that $w$ is a contractive fixed point of $f$ if $w$ attracts each point of $X ; w$ is a uniformly contractive fixed point if $w$ attracts $X ; w$ is a locally uniformly contractive fixed point if, for each $x$ in $X, w$ attracts some neighborhood of $x$. Clearly, the last two concepts are equivalent if $X$ is compact. For the general concept of attractor see $[12,5]$.

For a self-map $f$ on a metric space $(X, d), f$ is nonexpansive if

$$
d(f x, f y) \leq d(x, y) \text { for all } x, y \text { in } X \text {. }
$$

We say that $f$ is contractive if the inequality in (2) is strict for $x \neq y$ and $f$ is iteratively contractive if

$$
\text { given } x \neq y \text { there exists } r \text { in } N \text { with } d\left(f^{r} x, f^{r} y\right)<d(x, y) .
$$

Finally, $f$ is Banach contractive if there exists $\alpha<1$ such that

$$
d(f x, f y) \leq \alpha d(x, y) \text { for all } x, y \text { in } X .
$$

In [9] we showed that a necessary and sufficient condition for a contractive fixed point of a continuous self-map $f$ on a compact metric space $(X, d)$ is the equivalence of all orbits,

$$
d\left(f^{n} x, f^{n} y\right) \rightarrow 0 \text { as } n \rightarrow \infty \text { for all } x, y \text { in } X .
$$

Our main result here gives eleven characterizations (five of them new) of uniformly contractive fixed points for continuous self-maps on compact metric spaces. Example 1 in [13] shows that for such maps a contractive fixed point need not be uniformly contractive, contradicting some false results in the literature, e.g. Theorem 4.1 in [11]. (See also $[5,13]$.)

A basic result is Edelstein's Contraction Theorem [2, 9]: A contractive self-map on a compact metric space has a uniformly contractive fixed point. This result

Received by the editors August 12, 1981 and, in revised form, November 9, 1981.

1980 Mathematics Subject Classification. Primary 54H25; Secondary 54E45.

(c) 1982 American Mathematical Society 0002-9939/81/0000-0387/\$02:50 
breaks down if $f$ is only iteratively contractive and continuous. Such maps have unique fixed points which need not be contractive, let alone uniformly contractive. However, if $f$ is both nonexpansive and iteratively contractive, then $f$ has a uniformly contractive fixed point.

For $E$ a subset of $X$ let $d(E)$ be the diameter of $E$. For a countable set $E$ of points $x_{1}, x_{2}, \ldots$ we may denote $d(E)$ by $d\left(x_{1}, x_{2}, \ldots\right)$. See [7 or 13] for a definition of even continuity for a family of maps.

The following theorem will be proved in $\S 3$.

THEOREM 1. For a continuous self-map $f$ on a compact metric space $(X, d)$ the following conditions are equivalent.

$\left(1^{\circ}\right) f$ has a uniformly contractive fixed point $w$;

$\left(2^{\circ}\right) f$ is Banach contractive under some metric equivalent to $d$;

$\left(3^{\circ}\right) f$ is contractive under some metric equivalent to $d$;

$\left(4^{\circ}\right) d\left(f^{n} x, f^{n} y\right) \rightarrow 0$ uniformly for all $x, y$ in $X$

$\left(5^{\circ}\right)$ some point $w$ in $X$ attracts compact sets under $f$;

$\left(6^{\circ}\right)$ given $x$ in $X$ and $\epsilon>0$ there exists $r$ in $N$ such that $d\left(f^{r} x, f^{r} y\right)<\epsilon$ for all $y$ with $d(x, y) \leq \epsilon$

$\left(7^{\circ}\right)$ the core $Y=\bigcap_{n \in N} f^{n} X$ consists of a single point $w$;

$\left(8^{\circ}\right)$ given $\epsilon>0$ there exists $r$ in $N$ such that $d\left(f^{r} x, f^{r} y\right)<\epsilon$ for all $x, y$ with $d\left(x, y, f x, f y, f^{2} x, f^{2} y, \ldots\right)=\epsilon$

$\left(9^{\circ}\right)$ given $\epsilon>0$ there exists $r$ in $N$ such that $d(x, y)>\epsilon$ for all $x, y$ with $d\left(f^{r} x, f^{r} y\right)=\epsilon$;

$\left(10^{\circ}\right)$ given $u \neq v$ in $X$ there exists $r$ in $N$ such that $d(x, y)>d(u, v)$ for all $x, y$ with $f^{r} x=u, f^{r} y=v$;

$\left(11^{\circ}\right) f$ is iteratively contractive and the iterates of $f$ are equicontinuous;

$\left(12^{\circ}\right) f$ has a fixed point $w$ which is a limit point of every orbit, and the iterates of $f$ are evenly continuous.

2. Preliminary results. In this section we assemble some fundamental results to be used in the proof of Theorem 1.

PROPOSITION 1. Let $Y$ be a nonempty set in a compact metric space. Among all closed balls containing $Y$ there exists one of minimum radius $\epsilon \geq 0$.

Proof. Let $W$ consist of all $(x, z)$ such that $d(x, z) \geq d(x, y)$ for all $y$ in $Y$. Then $W$ is nonempty since $d(x, z)=d(X)$ for some $x, z$. Moreover, $W$ is closed in $X^{2}$. So $W$ is compact. Since $d$ is continuous on $X^{2}$, its restriction to the compact set $W$ attains a minimum $\epsilon$.

PROPOSITION 2 [9]. For a continuous self-map $f$ on a compact metric space $(X, d)$ the core $Y$ is compact, $d\left(f^{n} X\right) \downarrow d(Y)$ as $n \uparrow \infty$, and $f Y=Y$.

ProOF. The first two conditions hold for any nested sequence $Y_{n} \supset Y_{n+1}$ of compact sets. Indeed, continuity of diameter holds because $Y_{n}$ is ultimately contained in any given neighborhood of $Y$ since the sequence $\left\langle Y_{n} \cap C\right\rangle$ cannot have the finite intersection property for any compact $C$ disjoint from $Y$.

Given $y$ in $Y, y \in f^{n} X$ and so $f y \in f^{n+1} X$ for all $n$. That is, $f y \in Y$. So $f Y \subseteq Y$. To reverse this, $y \in f^{n+1} X$ for $y$ in $Y$ and all $n$. Pick $x_{n}$ in $f^{n} X$ with $f x_{n}=y$. The sequence $\left\langle x_{n}\right\rangle$ has a limit point $x$ in the compact space $X$. Since 
$f$ is continuous, $f x$ is a limit point of $\left\langle f x_{n}\right\rangle$. Since the latter is just the constant sequence $\langle y\rangle, f x=y$. Now $x_{n} \in f^{m} X$ for $n \geq m$, and $f^{m} X$ is closed. So any limit point $x$ of $\left\langle x_{n}\right\rangle$ belongs to $f^{m} X$ for all $m$, hence belongs to $Y$.

The next proposition is a variant of a result of G. Jungck [6].

Proposition 3. A continuous self-map $f$ on a compact metric space $(X, d)$ has a fixed point if, and only if, given $x \neq y$ there exists $h: X \rightarrow X$ such that $f h=h f$ and $d(h x, h y)<d(x, y)$.

Proof. Given $f w=w$ take $h x=w$ for all $x$. For the converse take $w$ with $d(w, f w)=\operatorname{Min}_{x} d(x, f x)$. Suppose $w \neq f w$. Get $h$ with $f h=h f$ and $d(h w, h f w)<d(w, f w)$. For $x=h w$ this inequality becomes $d(x, f x)<d(w, f w)$ contradicting our choice of $w$.

PROPOSITION 4 [8]. A continuous, iteratively contractive self-map $f$ on a compact metric space $(X, d)$ has a unique fixed point $w$ and

$$
\liminf _{n \rightarrow \infty} d\left(f^{n} x, f^{n} y\right)=0 \text { for all } x, y \text { in } X \text {. }
$$

So $w$ is a limit point of every orbit.

ProOF. The existence of a fixed point $w$ follows from Proposition 3 with $h=$ $f^{r}$. Uniqueness follows from (3). Suppose $\liminf d\left(f^{n} x, f^{n} y\right)=\epsilon>0$ for some $x, y$. Since $X^{2}$ is compact there exists a limit point $(u, v)$ of $\left\langle\left(f^{n} x, f^{n} y\right)\right\rangle$ with $d(u, v)=\epsilon$. Apply (3) to $u, v$ to get $r$ with $d\left(f^{r} u, f^{n} v\right)<\epsilon$. Since $(u, v)$ is a limit point of $\left\langle\left(f^{n} x, f^{n} y\right)\right\rangle$ so is $\left(f^{r} u, f^{r} v\right)$. Hence, by the definition of $\epsilon, \epsilon \leq d\left(f^{r} u, f^{r} v\right)$, a contradiction. So $\epsilon=0$ which gives (6). Apply (6) with $y=w$ to conclude that $w$ is a limit point of every orbit $\left\langle f^{n} x\right\rangle$.

PROPOSITION 5. Let $f$ be a continuous self-map on a Hausdorff space $X$ in which each point has a countable base of neighborhoods. For $w$ in $X$ conditions (a), (b), (c) are equivalent.

(a) $w$ is a locally uniformly contractive fixed point of $f$;

(b) $w$ attracts compact sets under $f$;

(c) (i) $f w=w$, (ii) given $x$ in $X$ and a neighborhood $B$ of $w, f^{m} x \in B$ for some $m$ in $N$, and (iii) the iterates of $f$ are evenly continuous.

Proof. Let (a) hold. Then a compact set $C$ is contained in a finite union of open sets which are attracted to $w$. So $w$ attracts $C$. Hence (b) holds.

Let (b) hold. Then (i) and (ii) are trivial. To prove (iii) consider any $x, y$ in $X$ and any neighborhood $D$ of $y$. Choose a nested base $A_{1} \supseteq A_{2} \supseteq \cdots$ of neighborhoods of $x$ and a similar base $B_{1} \supseteq B_{2} \supseteq \cdots$ for $y$. To prove (iii) we must show there exists $i$ such that $f^{n} A_{i} \subseteq D$ for all $n$ in $N$ with $f^{n} x$ in $B_{i}$. Suppose no such $i$ exists. Then for each $i$ in $N$ we can choose $n=n_{i}$ in $N$ and $x_{i}$ in $A_{i}$ such that for $y_{i}=f^{n} x$ and $z_{i}=f^{n} x_{i}, y_{i} \in B_{i}$ and $z_{i} \notin D$. We contend $y$ is a limit point of $\left\langle z_{i}\right\rangle$ giving the contradiction $z_{i} \in D$ for some $i$.

Case 1 . For some $n$ in $N, n_{i}=n$ for infinitely many $i$. Since $x_{i} \rightarrow x$ and $f^{n}$ is continuous, $f^{n} x$ is a limit point of $\left\langle z_{i}\right\rangle$. Since $y_{i} \rightarrow y$ and $y_{i}=f^{n} x$ for infinitely many $i, y=f^{n} x$. So $y$ is a limit point of $\left\langle z_{i}\right\rangle$.

Case 2. $n_{i} \rightarrow \infty$ as $i \rightarrow \infty$. Since $x_{i} \rightarrow x,\left\{x, x_{1}, x_{2}, \ldots\right\}$ is compact, hence is attracted by $w$. So $z_{i} \rightarrow w$ and $y_{i} \rightarrow w$. But $y_{i} \rightarrow y$. So $y=w$. Thus $z_{i} \rightarrow y$. 
Let (c) hold. To prove (a) we must show that given a neighborhood $D$ of $w$ and any $x$ in $X$ there exists a neighborhood $A$ of $x$ and $m$ in $N$ such that

$$
f^{m+n} A \subseteq D \text { for all } n \text { in } N \text {. }
$$

Since the iterates of $f$ are evenly continuous from $w$ to $w$ and $f^{n} w=w$ lies in every neighborhood of $w$, there exists a neighborhood $C$ of $w$ such that

$$
f^{n} C \subseteq D \text { for all } n \text { in } N \text {. }
$$

By even continuity from $x$ to $w$ there exist neighborhoods $A$ of $x$ and $B$ of $w$ such that

$$
f^{m} A \subseteq C
$$

for all $m$ with $f^{m} x$ in $B$. Thus (ii) implies (9) for some $m$. Then (7) follows from (8) and (9).

3. Proof of Theorem 1. We shall verify the following implications: $\left(5^{\circ}\right) \rightarrow$ $\left(1^{\circ}\right) \rightarrow\left(2^{\circ}\right) \rightarrow\left(3^{\circ}\right) \rightarrow\left(1^{\circ}\right) \rightarrow\left(4^{\circ}\right) \rightarrow\left(11^{\circ}\right) \rightarrow\left(12^{\circ}\right) \rightarrow\left(5^{\circ}\right),\left(4^{\circ}\right) \rightarrow\left(6^{\circ}\right) \rightarrow\left(7^{\circ}\right) \rightarrow$ $\left(4^{\circ}\right) \rightarrow\left(8^{\circ}\right) \rightarrow\left(7^{\circ}\right)$, and $\left(4^{\circ}\right) \rightarrow\left(9^{\circ}\right) \rightarrow\left(10^{\circ}\right) \rightarrow\left(7^{\circ}\right)$. The equivalence of $\left(1^{\circ}\right)$ and $\left(5^{\circ}\right)$ is trivial for compact spaces. $\left(1^{\circ}\right)$ implies $\left(2^{\circ}\right)$ for any metrizable space by Meyers' theorem [10]. $\left(2^{\circ}\right)$ implies $\left(3^{\circ}\right)$ a fortiori. $\left(3^{\circ}\right)$ implies $\left(1^{\circ}\right)$ by Edelstein's contraction theorem. The equivalence of $\left(1^{\circ}\right)$ and $\left(4^{\circ}\right)$ is trivial for any complete metric space. That $\left(4^{\circ}\right)$ implies $\left(11^{\circ}\right)$ is trivial. To prove $\left(11^{\circ}\right)$ implies $\left(12^{\circ}\right)$ apply Proposition 4 and note that equicontinuity implies even continuity. $\left(12^{\circ}\right)$ implies $\left(5^{\circ}\right)$ by Proposition 5. That $\left(4^{\circ}\right)$ implies $\left(6^{\circ}\right)$ is trivial.

To prove $\left(6^{\circ}\right)$ implies $\left(7^{\circ}\right)$ suppose the core $Y$ is nontrivial. Then $Y$ is contained in some closed ball of minimum radius $\epsilon>0$ by Proposition 1. Let $x$ be the center of such a ball $B$. Apply $\left(6^{\circ}\right)$ to get $r$ with $d\left(f^{r} x, f^{r} y\right)<\epsilon$ for all $y$ in $B$, hence for all $y$ in $Y$. Since $f^{r} Y=Y$ by Proposition 2, $d\left(f^{r} x, z\right)<\epsilon$ for all $z$ in $Y$. Hence, since $Y$ is compact, $Y$ is contained in a closed ball about $f^{r} x$ of radius less than $\epsilon$, contradicting the definition of $\epsilon$. So $d(Y)=0$ giving $\left(7^{\circ}\right) .\left(7^{\circ}\right)$ implies $\left(4^{\circ}\right)$ by Proposition 2. That $\left(4^{\circ}\right)$ implies $\left(8^{\circ}\right)$ is trivial. To prove $\left(8^{\circ}\right)$ implies $\left(7^{\circ}\right)$ suppose $Y$ is nontrivial. Let $\epsilon=d(Y)$. Get $r$ from $\left(8^{\circ}\right)$. Since $f Y=Y$ we can choose $x, y$ in $Y$ with $d\left(f^{r} x, f^{r} y\right)=\epsilon$. So

$$
\epsilon=d\left(f^{r} x, f^{r} y\right) \leq d\left(x, y, f x, f y, \ldots, f^{r} x, f^{r} y, \ldots\right) \leq d(Y)=\epsilon .
$$

This contradicts $\left(8^{\circ}\right)$.

$\left(4^{\circ}\right)$ implies $\left(9^{\circ}\right)$ with the conclusion of $\left(9^{\circ}\right)$ holding vacuously for $r$ large enough so that $d\left(f^{r} x, f^{r} y\right)<\epsilon$ for all $x, y$ in $X$. $\left(9^{\circ}\right)$ implies $\left(10^{\circ}\right)$ if we let $\epsilon=d(u, v)$. To prove $\left(10^{\circ}\right)$ implies $\left(7^{\circ}\right)$ suppose the core $Y$ is nontrivial. Get $u, v$ in $Y$ with $d(u, v)=d(Y)>0$. Apply $\left(10^{\circ}\right)$ to get $r$. Since $f^{r} Y=Y$ by Proposition 2 , there exist $x, y$ in $Y$ with $f^{r} x=u, f^{r} y=v$. For such $x, y\left(10^{\circ}\right)$ implies $d(x, y)>d(Y)$, a contradiction. So $d(Y)=0$ which gives $\left(7^{\circ}\right)$.

\section{Corollaries.}

COROLLARY 1. For a nonexpansive mapping $f$ on a compact metric space, the following are equivalent.

(a) $f$ has a uniformly contractive fixed point;

(b) $f$ has a contractive fixed point;

(c) $f$ is iteratively contractive. 
ProOF. (a) $\rightarrow$ (b) $\rightarrow$ (c) a fortiori. Since $\left(11^{\circ}\right)$ implies $\left(1^{\circ}\right)$ in Theorem 1, (c) implies (a) under (2).

COROLLARY 2. Let $f$ be a continuous self-map on a compact metric space $(X, d)$. Assume there exists $r$ in $N$ such that

$$
d\left(f^{r} x, f^{r} y\right)<d\left(x, y, f x, f y, f^{2} x, f^{2} y, \ldots\right) \quad \text { for all } x \neq y .
$$

Then $f$ has a uniformly contractive fixed point.

ProOF. $(10) \rightarrow\left(8^{\circ}\right) \rightarrow\left(1^{\circ}\right)$ by Theorem 1.

COROLlARY 3. Let $f$ be a continuous self-map on a compact metric space $(X, d)$ such that

$$
d(f x, f y)<d(x, y, f x, f y) \quad \text { for all } x \neq y .
$$

Then $f$ has a uniformly contractive fixed point.

PROOF. (11) implies (10) for $r=1$.

All three corollaries generalize Edelstein's contraction theorem. Corollary 3 subsumes a theorem of B. Fisher [3] who used the condition

$$
d(f x, f y)<\frac{1}{2}[d(x, f y)+d(y, f x)] \text { for } x \neq y
$$

which easily implies (11). Corollary 3 in turn can be shown to be subsumed by the following theorem of J. Danes [1]: Let $(X, d)$ be a complete metric space, $f$ a self-map on $X$, and $\phi:[0, \infty) \rightarrow[0, \infty)$ such that $\phi(s) \leq \phi(t)$ for $s \leq t, \phi(t+)<t$ for $t>0$, and

$$
d(f x, f y) \leq \phi[d(x, y, f x, f y)] \text { for all } x, y \text { in } X
$$

Then if some orbit is bounded there is a unqiue fixed point $w$ and all bounded orbits converge to $w$. For all bounded orbits $d\left(f^{n} x, f^{n+1} x, \ldots\right) \leq \phi^{n}\left[d\left(x, f x, f^{2} x, \ldots\right)\right]$.

Condition (10) with $r=1$ is a special case for a continuous self-map on a compact metric space of the "generalized Meir-Keeler contractions" of Hegedüs [4]. His definition of such maps is equivalent to the following relaxation of (13) given by Walter [14].

$$
d(f x, f y) \leq \phi\left[d\left(x, y, f x, f y, f^{2} x, f^{2} y, \ldots\right)\right] \text { for all } x, y
$$

with the assumption that all orbits are bounded. Walter, and in effect Hegedüs, generalizes Danes' theorem, replacing (13) by the weaker condition (14).

5. An example for Proposition 4. The unique fixed point of a continuous, iteratively contractive self-map on a compact metric space need not be a contractive fixed point.

Let $C$ be the closed subset of $[0,1]$ consisting of 0 and the reciprocals $\frac{1}{n}$ of all $n$ in $N$. Let $X$ consist of all $(x, y)$ in $C^{2}$ with $y \leq x$. Let $d$ be the Euclidean metric in the plane. $X$ is compact since it is the intersection of the compact set $C^{2}$ with a closed half plane. For $(x, y)$ in $X$ define $f(x, y)$ to be $(x /(1-x), y)$ for $x<1$ and $(y /(1+y), y /(1+y))$ for $x=1$. Clearly $f$ is continuous on each of the complementary subsets $x<1$ and $x=1$ of $X$. Since these sets are separated in $X, f$ is continuous. Clearly $(0,0)$ is the unique fixed point of $f$. Moreover, $f(1 /(n+1), 0)=\left(\frac{1}{n}, 0\right)$ and $f^{n}\left(\frac{1}{n}, 0\right)=(0,0)$ for all $n$ in $N$. The orbit of 
$(1,1)$ is composed of a succession $S_{1}, S_{2}, \ldots$ of finite sequences of the form $S_{n}=$ $\left\langle\left(\frac{1}{n}, \frac{1}{n}\right),\left(1 /(n-1), \frac{1}{n}\right), \ldots,\left(1, \frac{1}{n}\right)\right\rangle$ with $n$ terms. The orbit of $(1,1)$ includes every point $(x, y)$ in $X$ with $y>0$. Since $\left(\frac{1}{n}, \frac{1}{n}\right) \rightarrow(0,0)$ and $\left(1, \frac{1}{n}\right) \rightarrow(1,0)$ the orbit of $(1,1)$ is not convergent. We contend that $f$ is iteratively contractive.

Given any finite subset $A$ of $X$ and $\epsilon>0$ we shall find $r$ in $N$ such that $d\left(f^{r} A\right)<\epsilon$. For $A$ a two-point set and $\epsilon=d(A)$ this gives (3). Take $k$ large enough so that the following two conditions hold: (i) all $(x, y)$ in $A$ with $y>0$ are included in the first $k$ terms of the orbit of $(1,1)$, (ii) $f^{k}(x, 0)=(0,0)$ for all $(x, 0)$ in $A$. Take $n>k+\frac{2}{\epsilon}$ and set $r=n(n-1) / 2$. Since $n>k$ and $r=1+2+\cdots+n-1, f^{r}$ maps the first $k$ terms of the orbit of $(1,1)$ into the first $k$ terms of $S_{n}$. Let $B$ consist of $(0,0)$ and the first $k$ terms of $S_{n}:\left(\frac{1}{n}\right),\left(1 /(n-1), \frac{1}{n}\right), \ldots,\left(1 /(n-k+1), \frac{1}{n}\right)$. By (i) and (ii), $f^{r} A \subseteq B$ since $r \geq k$. Thus

$$
d\left(f^{r} A\right) \leq d(B)=\left[n^{-2}+(n-k+1)^{-2}\right]^{1 / 2}<\frac{2}{n-k+1}<\epsilon
$$

since $n>k+\frac{2}{\epsilon}$.

\section{REFERENCES}

1. J. Daner, Two fixed point theorems in topological and metric spaces, Bull. Austral. Math. Soc. 14 (1976), 259-265.

2. M. Edelstein, On fixed and periodic points under contractive mappings, J. London Math. Soc. 37 (1962), 74-79.

3. B. Fisher, $A$ fixed point theorem for compact metric spaces, Publ. Math. Debrecen 25 (1978), 193-194.

4. M. Hegedüs, New generalizations of Banach's contraction principle, Acta Sci. Math. (Szeged) 42 (1980), 87-89.

5. L. Janos, H.-M. Ko and K.-K. Tan, Edelstein's contractivity and attractors, Proc. Amer. Math. Soc. 76 (1979), 339-344.

6. G. Jungck, Commuting mappings and fixed points, Amer. Math. Monthly 83 (1976), 261-263.

7. J. L. Kelley, General topology, Van Nostrand, New York, 1955.

8. M. S. Khan and S. P. Singh, Some fixed point theorems for densifying mappings, Kyungpook Math. J. 20 (1980), 105-110.

9. S. Leader and S. L. Hoyle, Contractive fixed points, Fund. Math. 87 (1975), 93-108.

10. P. R. Meyers, A converse to Banach's contraction theorem, J. Res. Nat. Bur. Standards B 71 (1967), 73-76.

11. P. Morales, Topological contraction principle, Fund. Math. 110 (1980), 135-144.

12. R. Nussbaum, Some asymptotic fixed point theorems, Trans. Amer. Math. Soc. 171 (1972), 349-375.

13. J. L. Solomon and L. Janos, Even continuity and the Banach contraction principle, Proc. Amer. Math. Soc. 69 (1978), 166-168.

14. W. Walter, Remarks on a paper by F. Browder about contraction, Nonlinear Anal. 5 (1981), 21-25.

DEPARTMENT OF MAThematics, Rutgers UNIVERSity, NeW BRUNSWick, NeW JERSEY 08903 